Supporting Information

\title{
Cytosine Catalysis of Nitrosative Guanine Deamination and Interstrand Cross-Link Formation
}

Rainer Glaser*, Hong Wu, and Michael Lewis

Department of Chemistry, University of Missouri-Columbia, Columbia,Missouri 65211

glaserr@missouri.edu 
Complete Ref. 16: Wink, D. A.; Kasprzak, K. S.; Maragos, C. M.; Elespuru, R. K.; Misra, M.; Dunams, T. M.; Cebula, T. A.; Koch, W. H.; Andrews, A. W.; Allen, J. S.; Keefer, L. K. Science 1991, 254, 1001.

Complete Ref. 35: Frisch, M. J.; Trucks, G. W.; Schlegel, H. B.; Scuseria, G. E.; Robb, M. A.; Cheeseman, J. R.; Montgomery, J. A.; Vreven, Jr. T.; Kudin, K. N.; Burant, J. C.; Millam, J. M.; Iyengar, S. S.; Tomasi, J.; Barone, V.; Mennucci, B.; Cossi, M.; Scalmani, G.; Rega, N.; Petersson, G. A.; Nakatsuji, H.; Hada, M.; Ehara, M.; Toyata, K.; Fukuya, R.; Hasegawa, J.; Ishida, M.; Nakajima, T.; Honda, Y.; Kitao, O.; Nakai, H.; Klene, M.; Li, X.; Knox, J. E.; Hratchian, H. P.; Cross, J. B.; Adamo, C.; Jaramillo, J.; Gomperts, R.; Stratmann, R. E.; Yazyev, O.; Austin, A. J.; Cammi, R.; Pomelli, C.; Ochterski, J. W.; Ayala, P. Y.; Morokuma, K.; Voth, G. A.; Salvador, P.; Dannenberg, J. J.; Zakrzewski, V. G.; Dapprich, S.; Daniels, A. D.; Stain, M. C.; Farkas, O.; Malick, D. K.; Rabuck, A. D.; Raghavachari, K.; Foresman, J. B.; Ortiz, J. V.; Cui, Q.; Baboul, A. G.; Clifford, S.; Cioslowski, J.; Stefanov, B. B.; Liu, G.; Liashenko, A.; Piskorz, P.; Komaromi, I.; Martin, R. L.; Fox, D. J.; Keith, T.; Al-Laham, M. A.; Peng, C. Y.; Nanayakkara, A.; Challacombe, M.; Gill, P. M. W.; Johnson, B.; Chen, W.; Wong, M. W.; Gonzalez, C.; Pople, J. A. Gaussian, Inc., Pittsburgh PA, 2003. 
Table S1. Energetic and Thermodynamics Data at MP2(full)/6-31G*.

\begin{tabular}{|c|c|c|c|c|c|}
\hline Molecule & $\mathbf{E}$ & VZPE & TE & $\mathrm{S}$ & Nime \\
\hline 1 & -393.799136 & 62.64 & 66.75 & 80.15 & 0 \\
\hline 2 & -594.155162 & 60.51 & 66.16 & 93.64 & 0 \\
\hline 3 & -988.034511 & 124.05 & 134.53 & 134.26 & 0 \\
\hline 4 & -593.799289 & 51.74 & 57.48 & 94.58 & 0 \\
\hline 5 & -394.176909 & 71.25 & 75.42 & 80.37 & 0 \\
\hline 6 & -988.049698 & 124.94 & 135.39 & 134.39 & 0 \\
\hline 7 & -988.054093 & 122.62 & 134.58 & 151.57 & 0 \\
\hline 9 & -878.785471 & 118.87 & 127.95 & 127.27 & 1 \\
\hline$E-10$ & -484.583638 & 45.59 & 50.68 & 89.70 & 0 \\
\hline$Z-10$ & -484.581498 & 45.61 & 50.72 & 90.46 & 0 \\
\hline TS (10) & -484.552336 & 45.13 & 49.96 & 88.79 & 1 \\
\hline$E-11$ & -878.807437 & 117.90 & 127.71 & 132.69 & 1 \\
\hline$Z-11$ & -878.780796 & 117.39 & 127.33 & 131.93 & 1 \\
\hline $\mathbf{N}_{2}$ & -109.261574 & 3.12 & 4.60 & 45.87 & 0 \\
\hline \multicolumn{6}{|l|}{ Dediaz. $6 \rightarrow 7$} \\
\hline 6. $d(C N)=1.433$ & -988.049698 & 124.94 & 135.39 & 134.39 & 0 \\
\hline 1.6 & -988.045924 & & & & \\
\hline 1.8 & -988.043025 & & & & \\
\hline 2.0 & -988.045639 & & & & \\
\hline 2.2 & -988.049903 & & & & \\
\hline 2.4 & -988.052847 & & & & \\
\hline $7, \mathrm{~d}(\mathrm{CN})=2.684$ & -988.054093 & 122.62 & 134.58 & 151.57 & 0 \\
\hline \multicolumn{6}{|l|}{ Ring-open. $9 \rightarrow 11$} \\
\hline $\mathbf{9}, \mathrm{d}(\mathrm{CN})=1.494$ & -878.785849 & 118.87 & 127.95 & 127.27 & 1 \\
\hline 1.6 & -878.785545 & & & & \\
\hline 1.8 & -878.783900 & & & & \\
\hline 2.0 & -878.784674 & & & & \\
\hline 2.2 & -878.788874 & & & & \\
\hline 2.4 & -878.794716 & & & & \\
\hline 2.6 & -878.799681 & & & & \\
\hline 2.8 & -878.803291 & & & & \\
\hline 3.0 & -878.805629 & & & & \\
\hline 3.2 & -878.806900 & & & & \\
\hline 3.4 & -878.807304 & & & & \\
\hline 11, $d(C N)=3.544$ & -878.807437 & 117.90 & 127.71 & 132.69 & 1 \\
\hline
\end{tabular}


Cartesian Coordinates of Optimized Structures 1-11. 1

11

C $\quad-0.7190069929$

$\mathrm{N} \quad-1.3225886319$

$\mathrm{N} \quad-1.8051488596$

$\mathrm{N} \quad 0.5906083301$

C $\quad 1.0995258607$

$\mathrm{N} \quad 2.4171099812$

C $\quad 2.4660329763$

$\mathrm{N} \quad 1.2609531555$

0.4054755991

$-1.0416237901$

$-1.8221515627$

$-1.5325005289$

3.2050159679

3.4000599216

$-2.5493194309$

$-1.2517573636$

$-2.5048196481$

$-3.5393522891$

$-1.2984262547$

0.0557122057

0.2937647137

1.6553374233

2. 2213153231

1.1793910392

1.2090013469

2.1246840836

$-0.1742513583$

$-0.3457127231$

2.2012830977

$-0.2582178899$
$-0.0067798948$

$-0.0045951738$

$-0.0014675309$

0.0973405051

0.0881108602

0.1772780862

0.1273953084

0.0109120408

$-0.0148342687$

$-0.1290596848$

$-0.2261435042$

$-0.1128672536$

0.2640609621

0.1788419827

$-0.1889535486$
2

01

$\mathrm{N} \quad-0.8919037609$

C $\quad-0.8765664483$

C $\quad 0.3150319158$

C $\quad 1.4893942062$

$\mathrm{N} \quad 1.476968047$

0.2952522193

$-2.0896520715$

$-2.1613754896$

$-2.8847090577$

0.287967564

2.4622502642

2.3383755603

0.3938667873

3

11

C

$\mathrm{N}$

$\mathrm{N}$

$\mathrm{N}$

C

N

C

N

C

C

O

$\mathrm{N}$

$\mathrm{H}$

$\mathrm{H}$

$\mathrm{H}$

$\mathrm{N}$
1.3065350529

0.6293016114

0.2711558682

2.6044055681

3. 2384262185

4.5824219341

4.7544582602

3.6114856343

2.6619168224

1. 2280371897

0.5655979834

0.5931387626

5.3060738607

5.734477686

$-0.497502856$

$-2.3931300504$
0.002607586

$-0.0041999779$

$-0.0034479077$

0.0065729446

0.0098541269

0.0019951075

$-0.0726629251$

0.2746509732

0.1117870903

$-0.0236654817$

0.008595025

0.0157122112

0.0036009585

$-1.2188385977$

$-2.4566570345$

$-3.529567898$

$-1.4133312542$

$-0.2241749836$

$-0.0009483598$

1.3544219569

2.0281743809

1.0605585398

1.2009070974

2.2254715883

$-0.0849137124$

$-0.7109214038$

1.8091380129

$-0.0248607589$

2.4426530609
$-0.5634649855$

0.7532571795

1.5530131237

0.8718306613

$-0.4846977727$

$-1.2661727601$

1.3835175027

2.3299889809

0.7828490559

2.6354584298

1.353581231

$-1.0203426538$

$-2.4875734352$

$-0.1003229386$

$-0.2078413894$

$-0.3170157519$

$-0.0114061129$

0.0219689643

0.1262590487

0.103648886

$-0.0106863884$

$-0.0617745928$

$-0.1841827168$

$-0.2761650726$

$-0.1874332169$

0.2051694179

0.1735237306

$-0.1641422779$

$-0.384678747$ 


$\begin{array}{ll}\mathrm{C} & -2.9301157716 \\ \mathrm{~N} & -2.099533928 \\ \mathrm{C} & -2.5528043958 \\ \mathrm{O} & -1.7795797556 \\ \mathrm{~N} & -3.9176288684 \\ \mathrm{C} & -4.7919498897 \\ \mathrm{C} & -4.3414354083 \\ \mathrm{H} & -1.3767179689 \\ \mathrm{H} & -2.9612430954 \\ \mathrm{H} & -4.2393124005 \\ \mathrm{H} & -5.8389905364 \\ \mathrm{H} & -5.0246547052\end{array}$

$0 \quad 1$

$\begin{array}{lr}\mathrm{N} & -3.5844942843 \\ \mathrm{~N} & -2.5488715485 \\ \mathrm{C} & -1.2397369616 \\ \mathrm{~N} & -1.3664257327 \\ \mathrm{C} & -0.1687909919 \\ \mathrm{O} & -0.1676369909 \\ \mathrm{C} & 0.9696330253 \\ \mathrm{C} & 0.8151173992 \\ \mathrm{~N} & -0.3269231121 \\ \mathrm{~N} & 2.2703710087 \\ \mathrm{C} & 2.8883760423 \\ \mathrm{~N} & 2.0582893315 \\ \mathrm{H} & 3.9394518497 \\ \mathrm{H} & 2.3004333591\end{array}$

1. 2531943514

0.2067582656

$-1.041783236$

$-2.0086419639$

$-1.1924956356$

$-0.1513499787$

1.0950185517

2. 5266183864

3. 2776073562

$-2.128672922$

$-0.380454243$

1.9314836801
-0.1093013974
-0.0228135783
0.2800998476
0.379305607
0.4732887857
0.3704827986
0.0760916479
-0.4314300795
-0.4261158733
0.7011153542
0.5367340394
-0.0039501272
$-0.7194682313$

$-0.5118809923$

$-0.2514563822$

$-0.3230477425$

$-0.0897818592$

$-0.1310386021$

0.1863302224

0.2005926042

$-0.0224229405$

0.4561044303

0.6294695893

0.4870428495

0.8611000613

0.5719920962
1.573143523

1.1586351054

0.5012834471

0.7903265708

$-1.5508837851$

$-2.7718923041$

$-0.6769546552$

0.7183307804

1. 4277228651

$-1.0221207136$

0.1362823837

1. 2181218017

0.2521592914

2. 1983980407
5

11

$\begin{array}{cc}\mathrm{N} & 0.6950053743 \\ \mathrm{C} & -0.5645862003 \\ \mathrm{C} & 1.1238559523 \\ \mathrm{C} & -1.569928094 \\ \mathrm{~N} & 0.076121774 \\ \mathrm{O} & 2.2724448631 \\ \mathrm{~N} & -0.8156598854 \\ \mathrm{C} & -1.2045941315 \\ \mathrm{H} & 1.4566582746 \\ \mathrm{H} & -2.5951979965 \\ \mathrm{H} & 0.3343818835 \\ \mathrm{H} & -0.0990118244 \\ \mathrm{H} & -1.7554442873 \\ \mathrm{H} & -1.9177009537\end{array}$

0.6083655716

1.0701294719

$-0.7313046642$

0.1357885962

$-1.5756953416$

$-1.06909551$

2. 3618877907

$-1.1676456317$

1.2373872856

0.4494522439

$-2.5541166654$

3.0330258652

2. 7223343369

$-1.9390317642$
0.2402828703

0.0736389194

0.0938137931

$-0.2832436972$

$-0.2558935319$

0.2600296141

0.2465783115

$-0.4358849571$

0.4970989825

$-0.429572842$

$-0.3736096336$

0.5018496685

0.1249828997

$-0.7077038862$
6

11

$\begin{array}{ll}\mathrm{N} & 0.645239797 \\ \mathrm{~N} & 1.0597706116 \\ \mathrm{C} & 1.5925870831 \\ \mathrm{~N} & 0.674340049 \\ \mathrm{C} & 1.1171289349\end{array}$
$-2.890233165$

$-1.955663144$

$-0.778872042$

$-0.0434149375$

1.1306522083
$-1.8682575209$

$-1.37493086$

$-0.755678602$

$-0.1461574278$

0.4874730054 


$\begin{array}{ll}\mathrm{O} & 0.3237867221 \\ \mathrm{C} & 2.5396897948 \\ \mathrm{C} & 3.3283004061 \\ \mathrm{~N} & 2.9000551409 \\ \mathrm{~N} & 3.3044534442 \\ \mathrm{C} & 4.5343765234 \\ \mathrm{~N} & 4.6032211297 \\ \mathrm{H} & 5.4144374367 \\ \mathrm{H} & 5.4323062826 \\ \mathrm{~N} & -2.1865052602 \\ \mathrm{C} & -2.6344014908 \\ \mathrm{O} & -1.8873986092 \\ \mathrm{~N} & -4.0000782377 \\ \mathrm{C} & -4.8301683572 \\ \mathrm{C} & -4.3580992083 \\ \mathrm{C} & -2.9582107058 \\ \mathrm{~N} & -2.3768056609 \\ \mathrm{H} & -1.1551599341 \\ \mathrm{H} & -4.3605285759 \\ \mathrm{H} & -5.8794467327 \\ \mathrm{H} & -5.0207968124 \\ \mathrm{H} & -2.9293631896 \\ \mathrm{H} & -1.345608354\end{array}$

7

1

$\mathrm{N}$

C

C

O

C

C

$\mathrm{N}$

$\mathrm{N}$

C

$\mathrm{N}$

$\mathrm{H}$

$\mathrm{H}$

$\mathrm{N}$

C

C

C

$\mathrm{N}$

$\mathrm{N}$

C

O

$\mathrm{H}$

$\mathrm{H}$

$\mathrm{H}$

$\mathrm{H}$

$\mathrm{H}$

$\mathrm{H}$
1

$$
\begin{array}{r}
-0.2740700954 \\
0.2432037632 \\
1.5767279462 \\
0.7300487399 \\
1.4694938974 \\
0.8205259211 \\
2.8856531386 \\
3.4369894113 \\
2.7279808934
\end{array}
$$

3. 8742935422

4.990078926

4.7767861996

5.9861097845

5.4761169374

$-4.3294632537$

$-4.928854312$

$-4.2055131769$

$-2.789255772$

$-1.990236342$

$-2.2492578772$

$-2.9569528563$

$-2.4133649416$

$-4.8785637804$

$-6.0104742955$

$-4.6883772903$

$-2.3811669378$

$-0.9669477816$

$-1.2291866743$

$$
\begin{array}{r}
1.8787146758 \\
1.3478918784 \\
0.4154387808 \\
-0.7111405038 \\
2.3679098447 \\
2.0666645435 \\
0.9034428676 \\
2.6627234748 \\
0.4792008434 \\
-0.3042330947 \\
-1.4631299521 \\
-2.2236961338 \\
-1.6604495983 \\
-0.8070116039 \\
0.3162862383 \\
0.5845813978 \\
1.6361322997 \\
-0.1597905643 \\
-2.4999619411 \\
-1.08111008 \\
0.9905306303 \\
2.3154509305 \\
1.7813456933
\end{array}
$$

1.0823966391

0.366289279

$-0.3397118812$

$-0.947098001$

0.8490154261

0.4480484858

$-0.2704762228$

0.6533239691

$-0.6754605867$

0.2043797391

$-0.4188713748$

$-1.0141748185$

$-0.296471698$

0.3688925119

0.966798509

0.8807515559

1. 4153819218

0.1085923776

$-0.7422119182$

0.3887853949

1. 4936319203

1. 9257799722

1.3201378763
2. 9691563809

2. 3125114215

0.7969381645

0.147470796

$-0.7175806895$

$-1.443334941$

$-0.4916447601$

0.4069577363

1.1858862565

$-1.0802352262$

$-0.5486673225$

0.3593412303

$-0.7756554222$

0.8928470185

0.2273826854

$-0.6146496843$

$-1.3327245056$

$-1.1806982428$

$-1.8285548382$

$-0.3266347266$

0.4316977936

1. 1805857301

0.7607503351

$-0.6765389663$

$-2.0014882584$

$-2.4706600062$

$-1.7104843269$

$-0.1788154813$
$-3.217231347$

$-2.4571062564$

$-0.6873763313$

$-0.0687534952$

0.9620933997

1. 6884134502

0.8672968062

$-0.0577785279$

$-0.9869372553$

1. 6203385851

1. 1588168058

0.1401940774

1. 5158378705

$-0.3686759311$

$-0.7862365442$

0.0976029227

0.9963318926

0.9902080717

1.8172319486

0.087294213

$-0.8565165751$

$-1.6424334467$

$-1.4561838992$

0.0404007693

1. 6968014568

2.4970444009

1. 7967143193

0.0445927166 


$\begin{array}{lrll}9 & & & \\ 1 & 1 & & \\ \mathrm{~N} & -0.0001446962 & 0 . & -2.2387103797 \\ \mathrm{H} & 0.0213089365 & 0 . & -1.2073016422 \\ \mathrm{C} & 1.1791467501 & 0 . & -2.9008566761 \\ \mathrm{~N} & 2.3032245071 & 0 . & -2.2001529409 \\ \mathrm{H} & 2.2838943251 & 0 . & -1.1803422649 \\ \mathrm{H} & 3.1993359105 & 0 . & -2.6716668342 \\ \mathrm{C} & 1.1506077681 & 0 . & -4.3229587287 \\ \mathrm{H} & 2.066282447 & 0 . & -4.8995235991 \\ \mathrm{C} & -0.0657349097 & 0 . & -4.9316101571 \\ \mathrm{H} & -0.1740382816 & 0 . & -6.0112010783 \\ \mathrm{~N} & -1.2191051042 & 0 . & -4.2144177527 \\ \mathrm{H} & -2.1239498751 & 0 . & -4.6793480022 \\ \mathrm{C} & -1.2757594544 & 0 . & -2.8221737147 \\ \mathrm{O} & -2.3034055366 & 0 . & -2.1792470959 \\ \mathrm{O} & 1.0943957166 & 0 . & 0.3735715135 \\ \mathrm{C} & 0.2986838499 & 0 . & 1.3027416746 \\ \mathrm{~N} & -1.1653279353 & 0 . & 0.9965363191 \\ \mathrm{C} & -1.7794346807 & 0 . & 2.0586896996 \\ \mathrm{~N} & -1.9305473987 & 0 . & 3.3039687764 \\ \mathrm{C} & -0.5632807885 & 0 . & 3.6327650258 \\ \mathrm{C} & 0.5110528819 & 0 . & 2.7252815382 \\ \mathrm{~N} & 1.7111015871 & 0 . & 3.3966345722 \\ \mathrm{C} & 1.3680060653 & 0 . & 4.6707948455 \\ \mathrm{H} & 2.057537187 & 0 . & 5.5049015167 \\ \mathrm{~N} & 0.0007249578 & 0 . & 4.8651376686 \\ \mathrm{H} & -0.4974984052 & 0 . & 5.7508716807 \\ & & & \end{array}$

$\begin{array}{lrrr}\begin{array}{l}E-10 \\ 0\end{array} & & & \\ \mathrm{C} & -0.9249912968 & -1.5571754511 & 0.6552094925 \\ \mathrm{~N} & -1.8889638954 & -0.8907702286 & 0.3272281438 \\ \mathrm{C} & -1.4405722718 & 0.4589152297 & -0.2529355784 \\ \mathrm{O} & -2.2422826024 & 1.262161727 & -0.6357226661 \\ \mathrm{C} & 0.0130477108 & 0.4702236274 & -0.2071046864 \\ \mathrm{C} & 0.7736370656 & -0.5972619146 & 0.2908311192 \\ \mathrm{~N} & 0.3083559761 & -1.8160053077 & 0.812758924 \\ \mathrm{~N} & 0.8316534257 & 1.4946972367 & -0.6305858935 \\ \mathrm{C} & 2.0523478957 & 1.0553097746 & -0.3949530547 \\ \mathrm{~N} & 2.0706968077 & -0.2053535249 & 0.1632667367 \\ \mathrm{H} & 2.9652303107 & 1.5978732056 & -0.6030264276 \\ \mathrm{H} & 2.8800196879 & -0.7532118464 & 0.4338486261\end{array}$

$\begin{array}{lllr}Z-10 & & & \\ 0 & & & \\ \mathrm{~N} & 0.914599865 & -3.3471753066 & 0.9594340904 \\ \mathrm{C} & 1.0091978756 & -2.2081922313 & 0.6333283868 \\ \mathrm{~N} & 1.140694111 & -0.9261160142 & 0.2671098264 \\ \mathrm{C} & 0.0117879771 & -0.2916993769 & 0.0831678838 \\ \mathrm{~N} & -1.2884508976 & -0.7372436642 & 0.2076187967 \\ \mathrm{C} & -2.1520068648 & 0.3026832156 & -0.0912857386\end{array}$




$\begin{array}{lrcl}\mathrm{N} & -1.5547474679 & 1.4167501377 & -0.4085672063 \\ \mathrm{C} & -0.1902586723 & 1.0826655816 & -0.310036617 \\ \mathrm{C} & 0.8009986415 & 1.957908964 & -0.5578687993 \\ \mathrm{O} & 1.6744557872 & 2.7009252096 & -0.7681979614 \\ \mathrm{H} & -1.5318625406 & -1.6855514638 & 0.479549782 \\ \mathrm{H} & -3.2267667741 & 0.1744467992 & -0.0569653364\end{array}$

TS (10)

01

$\begin{array}{cc}\mathrm{N} & 2.2133359127 \\ \mathrm{C} & 1.372006979 \\ \mathrm{~N} & 0.4648408471 \\ \mathrm{C} & -0.3784651064 \\ \mathrm{~N} & -1.7067681902 \\ \mathrm{C} & -2.3241154308 \\ \mathrm{~N} & -1.5321433728 \\ \mathrm{C} & -0.315807067 \\ \mathrm{C} & 0.8140793954 \\ \mathrm{O} & 1.8014443057 \\ \mathrm{H} & -2.1232707604 \\ \mathrm{H} & -3.3693326835\end{array}$

$-2.8108027365$

$-0.3765049941$

$-2.0818601895$

0.0470682723

$-1.3016724544$

๑. 5822876375

$-0.4296156779$

$\odot .1366164288$

$-0.6434860261$

$-0.1866786635$

๑. 5583696942

$-0.4635765976$

1. 593618797

$-0.3566268171$

1. 0118032998

○. 0201477637

1. 7214269465

๑. 232633306

2. 3009672764

$\odot .4336852136$

$-1.5693965571$

$-0.2036863822$

0. 6173108475

$-0.7404705051$

$\begin{array}{llll}\text { E-11 } & & & \\ \mathrm{O} & 1 & & \\ \mathrm{~N} & -0.801629 & 0 & -2.676334 \\ \mathrm{H} & -0.801629 & 0 & -1.646955 \\ \mathrm{C} & 0.392703 & 0 & -3.311989 \\ \mathrm{~N} & 1.490349 & 0 & -2.573832 \\ \mathrm{H} & 1.40955 & 0 & -1.551528 \\ \mathrm{H} & 2.403804 & 0 & -3.010236 \\ \mathrm{C} & 0.394372 & 0 & -4.734374 \\ \mathrm{H} & 1.321743 & 0 & -5.291838 \\ \mathrm{C} & -0.809483 & 0 & -5.367631 \\ \mathrm{H} & -0.895462 & 0 & -6.449204 \\ \mathrm{~N} & -1.978284 & 0 & -4.675308 \\ \mathrm{H} & -2.872914 & 0 & -5.159518 \\ \mathrm{C} & -2.064441 & 0 & -3.284519 \\ \mathrm{O} & -3.103958 & 0 & -2.661231 \\ \mathrm{~N} & 0.255544 & 0 & 0.077179 \\ \mathrm{C} & -0.235774 & 0 & 1.163836 \\ \mathrm{~N} & -0.865088 & 0 & 2.312789 \\ \mathrm{C} & -0.142481 & 0 & 3.418749 \\ \mathrm{~N} & -0.704949 & 0 & 4.660098 \\ \mathrm{H} & -1.709364 & 0 & 4.815241 \\ \mathrm{C} & 0.293812 & 0 & 5.61894 \\ \mathrm{H} & 0.068916 & 0 & 6.677777 \\ \mathrm{~N} & 1.497352 & 0 & 5.116245 \\ \mathrm{C} & 1.26367 & 0 & 3.731137 \\ \mathrm{C} & 2.287892 & 0 & 2.846967 \\ \mathrm{O} & 3.171541 & 0 & 2.091695 \\ & & & \end{array}$




\begin{tabular}{|c|c|c|c|}
\hline \multicolumn{4}{|c|}{$\begin{array}{l}Z-11 \\
11\end{array}$} \\
\hline C & -2.4695612321 & 1.8156505375 & \\
\hline $\mathrm{N}$ & -3.8124418496 & 2.2313654769 & \\
\hline C & -4.4650164134 & 1.1050540933 & \\
\hline $\mathrm{N}$ & -3.6495467461 & -0.0192820372 & \\
\hline C & -2.3394462634 & ๑. 3755740038 & \\
\hline $\mathrm{N}$ & -1.2505863531 & -0.3754319725 & \\
\hline C & -1.567133966 & -1.6905957772 & \\
\hline $\mathrm{N}$ & -1.9010435529 & -2.8295692251 & \\
\hline C & -1.4226538081 & 2.6513724314 & \\
\hline 0 & -0.4540463636 & 3.3080305413 & \\
\hline $\mathrm{H}$ & -5.5446768342 & 1.0209694206 & \\
\hline $\mathrm{H}$ & -3.9451284856 & -0.9944632559 & \\
\hline $\mathrm{N}$ & 1. 7816471871 & -0.5513112452 & \\
\hline C & 2.0772022473 & -1.9314895917 & \\
\hline 0 & 1.2250708727 & -2.7902987323 & \\
\hline $\mathrm{N}$ & 3.4476375328 & -2.1854784669 & \\
\hline C & 4.4025610955 & -1.2251202362 & \\
\hline C & 4.0755233318 & ๑. . 0959901136 & \\
\hline C & 2.6977447437 & 0.4382764966 & \\
\hline $\mathrm{N}$ & 2.2942420568 & 1.7048940664 & \\
\hline $\mathrm{H}$ & $\odot .7604367163$ & -0.3498617759 & \\
\hline $\mathrm{H}$ & 3.6955289784 & -3.1729827369 & \\
\hline $\mathrm{H}$ & 5.4315882268 & -1.5694005265 & \\
\hline $\mathrm{H}$ & 4.8394717374 & ๑. 8622684709 & \\
\hline $\mathrm{H}$ & 2.9723055869 & 2.4574210626 & \\
\hline $\mathrm{H}$ & 1.3176016645 & 1. 9696162671 & \\
\hline
\end{tabular}

